\title{
Adequate and Well-controlled Study
}

National Cancer Institute

\section{Source}

National Cancer Institute. Adequate and Well-controlled Study. NCI Thesaurus. Code C142382.

A controlled clinical study with the characteristics specified in Title 21, Section 314.126 of the FDA Code of Federal Regulations, including: a clear statement of the objectives of the investigation and a summary of the proposed methods of analysis in the protocol; a design that permits a valid comparison with a control to provide a quantitative assessment of the effect; a method of subject selection that provides adequate assurance that the subject has the disease or condition that the treatment is directed at; a method of assigning patients to treatment and control groups that minimizes bias and assures comparability of the groups; adequate measures taken to minimize bias, by the subjects, observers and the data analysts; methods of assessment of subjects' response that are well-defined and reliable; and an analysis of the results of the study that is adequate to assess the effect of the drug or device. 